\title{
Wireless-Powered Mobile Edge Computing with Cooperated UAV
}

\author{
(Invited Paper)
}

\author{
Xiaoyan $\mathrm{Hu}^{\dagger}$, Kai-Kit Wong ${ }^{\dagger}$ and Zhongbin Zheng ${ }^{\S}$ \\ ${ }^{\dagger}$ Department of Electronic and Electrical Engineering, University College London, London, UK \\ ${ }^{\S}$ East China Institute of Telecommunications, Shanghai, P. R. China \\ Email: ${ }^{\dagger}\left\{\right.$ xiaoyan.hu.16, kai-kit.wong\}@ucl.ac.uk, ${ }^{\S}$ ben@ecit.org.cn
}

\begin{abstract}
In this paper, we study a wireless-powered mobile edge computing (MEC) system, where the access point (AP) cooperates with an unmanned aerial vehicle (UAV). The AP broadcasts energy to the UAV, while the UAV broadcasts part of its harvested energy to the UEs and helps the UEs compute their offloaded tasks or further offload to the AP for computing. The weighted sum completed task-input bits (WSCTB) of UEs is maximized by optimizing the task allocation, the UAV's energy transmit power and trajectory, under the information-causality constraints, the energy-causality constraints, and the UAV's trajectory constraints. The formulated WSCTB maximization problem is non-convex, and a block coordinate descending algorithm is proposed to solve it iteratively. In the simulation results, the UAV's trajectory and the achieved performance are given to verify the effectiveness of the proposed algorithm in comparison with some practical baselines.
\end{abstract}

Index Terms-Mobile edge computing, UAV, wireless power transfer, task allocation, trajectory optimization.

\section{INTRODUCTION}

The blowout growth of intelligent mobile devices in accompany with various computation-intensive applications have driven the computing requirement of these devices increasing dramatically. In this case, the resource-limited mobile user equipment (UEs) are not always capable of handling the computation tasks related to these applications. To deal with this issue, the mobile edge computing (MEC) has emerged as a promising technology to provide cloud computing services for UEs at the edge of communication networks [1], e.g., at the access points (APs). The energy efficiency and latency are two important criteria for MEC works [2-5], where the energy computation or the latency (or the cost including both the energy and latency) for completing the UEs' computation tasks is minimized by jointly optimizing the radio and computing resource allocation. However, the traditional battery-based UEs cannot take full advantage of the edge computing when their energy is running out, and thus the technology of wireless power transfer (WPT) has been leveraged in a mass of works [6-8] to improve the efficiency of MEC.

For the conventional MEC architectures, the AP plays a vital role in helping the UEs compute their offloaded tasks. In order to relieve the burden of the AP for computing and improve users' experience, the unmanned aerial vehicle (UAV)-enabled MEC has drawn great attention [9-11], mainly due to the attractive advantages of the UAVs, such as flexible movement, line-of-sight (LoS) connections, and moderate computing and caching capabilities, etc. [12]. Similarly, the WPT has also been leveraged to improve the efficiency of UAV-enabled
MEC [13]. However, most of the existing UAV-enabled MEC works only rely on the UAV to compute UEs' offloaded task. Actually, the cooperation among the AP and the UAV is sometimes necessary since the AP can not always provide good connections to some edge users and the size-constrained UAV may lack of resources. In this paper, a wireless-powered UAVassisted MEC architecture is studied to explore the advantages of cooperation between the UAV and the AP.

\section{System Model And Problem Formulation}

A wireless-powered UAV-assisted MEC system is considered in this work, which consists of an AP, a cellular-connected UAV, and $K$ ground UEs, all being equipped with single antenna. The AP broadcasts radio frequency (RF) energy to the UAV during the task completion time. Part of UAV's harvested energy will be further broadcasted to the UEs, and the remaining part will be used for computing and transmissions. Suppose that each UE has a large mount of bit-wiseindependent computation task-input data, and the UAV acts as a MEC server as well as a relay to help the UEs compute their task-input data or further offload their data to the more powerful server at the AP for computing. It is assumed that the energy and the task information transmissions are operated simultaneously over orthogonal frequency bands, while the different kinds of task information transmissions are operated over the same frequency band, with the bandwidth $B$. In this paper, we assume the direct links between the UEs and the AP are negligible due to, e.g., severe blockage, and thus the UEs cannot directly harvest energy from the AP or offload their task-input bits to the AP unless with the help of the UAV.

\section{A. Node Locations and the Channel Model}

In this paper, we consider a scenario where the UAV flies at a fixed altitude, denoted as $H$, during the task completion time. The locations of the AP and all the UEs are fixed on the ground with zero altitude. Hence, we adopt a two-dimensional (2D) Euclidean coordinate to represent the horizontal locations of the UEs, the AP and the UAV, whose coordinates are measured in meters. The AP is fixed at the origin of the coordinate, i.e., $(0,0)$. Let $\mathcal{K}=\{1, \ldots, K\}$ denote the set of the UEs, and $\mathbf{v}_{k}=\left(x_{k}, y_{k}\right)$ is the horizontal location of UE $k \in \mathcal{K}$. The initial and final horizontal locations of the UAV are assumed to be preset as $\mathbf{u}_{\mathrm{I}}=\left(x_{\mathrm{I}}, y_{\mathrm{I}}\right)$ and $\mathbf{u}_{\mathrm{F}}=\left(x_{\mathrm{F}}, y_{\mathrm{F}}\right)$, respectively. The total task completion time $T$ is equally divided into $N$ time slots with $T=N \tau$, where $\tau$ is the slot duration and chosen to be sufficiently small to 


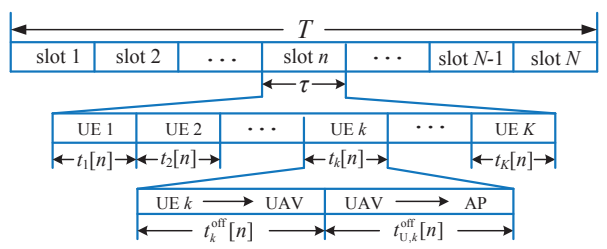

Fig. 1. The TDMA structure of the whole task completion time $T$.

guarantee an approximately unchanged UAV's location during each slot. Let $\mathcal{N}=\{1, \cdots, N\}$ denote the set of the $N$ time slots. At the $n$-th time slot, the UAV's horizontal location can be further denoted as $\mathbf{u}[n]=\mathbf{u}(n \tau)=(x[n], y[n])$ with $\mathbf{u}[0]=\mathbf{u}_{\mathrm{I}}$ and $\mathbf{u}[N]=\mathbf{u}_{\mathrm{F}}$, which should satisfy the maximum speed constraint, i.e., $v[n]=\|\mathbf{u}[n]-\mathbf{u}[n-1]\| / \tau \leq v_{\max }$ with $v_{\max }$ as UAV's predetermined maximum speed.

The wireless channels between UE $k \in \mathcal{K}$ and the UAV as well as that between the UAV and the AP are assumed to be dominated by LoS links. Thus, the corresponding channel power gains at time slot $n$ can be respectively given as

$$
\begin{aligned}
& h_{k}[n]=h_{0} d_{k}^{-2}=\frac{h_{0}}{\left\|\mathbf{u}[n]-\mathbf{v}_{k}\right\|^{2}+H^{2}}, \quad k \in \mathcal{K}, n \in \mathcal{N}, \\
& h_{\mathrm{AP}}[n]=h_{0} d_{\mathrm{AP}}^{-2}=\frac{h_{0}}{\|\mathbf{u}[n]\|^{2}+H^{2}}, n \in \mathcal{N},
\end{aligned}
$$

where $h_{0}$ is the channel power gain at a reference distance of $d_{0}=1 \mathrm{~m} ; d_{k}$ and $d_{\mathrm{AP}}$ are the horizontal plane distances between the UAV and UE $k$ as well as the AP at time slot $n$.

\section{B. Computing Methods for UEs' Task-Input Data}

UEs' computation tasks are composed of a large amount of task-input data (bits), and we use $C_{k}$ to represent the number of required CPU cycles for computing 1-bit of UE $k$ 's input data. ${ }^{1}$ Note that the UEs' task-input data are bit-wise independent and can be arbitrarily divided to facilitate parallel computation in three ways: local computing at UEs, data offloaded to the UAV for computing, and data further offloaded to the AP for computing. In order to avoid interference among the UEs during offloading when using the later two computing methods, we adopt the time-division multiple access (TDMA) protocol as shown in Fig. 1. Each slot $n \in \mathcal{N}$ is divided into $K$ durations, and the operations related to UE $k \in \mathcal{K}$ are all executed in the $k$-th duration $t_{k}[n] \in[0, \tau]$. The $t_{k}[n]$ duration is then be equally divided into two parts, one for UE $k$ 's offloading to the UAV, the other for UAV's further offloading to the AP, denoted as $t_{k}^{\text {off }}[n]$ and $t_{\mathrm{U}, k}^{\text {off }}[n]$, respectively. Next, we will give some details of the three computing methods.

1) Local Computing at the UEs: Local computing at the UEs can be performed simultaneously with wireless transmissions. Denoting the CPU frequency of UE $k$ during time slot $n$ as $f_{k}[n]$ (cycles/second), the computation bits and the energy consumption of UE $k$ during slot $n$ can be expressed as

$$
L_{k}^{\text {loc }}[n]=\tau f_{k}[n] / C_{k}, k \in \mathcal{K}, n \in \mathcal{N},
$$

\footnotetext{
${ }^{1}$ In our considered scenario, we assume that the output data sizes of the computation tasks are quite small that can be ignored especially compared with the input data sizes of the tasks.
}

$$
E_{k}^{\mathrm{loc}}[n]=\tau \kappa_{k} f_{k}^{3}[n] \equiv \frac{\kappa_{k} C_{k}^{3}}{\tau^{2}}\left(L_{k}^{\mathrm{loc}}[n]\right)^{3}, k \in \mathcal{K}, n \in \mathcal{N},
$$

where $\kappa_{k}$ is the effective capacitance coefficient of UE $k$ [14].

2) Data Offloaded to the UAV for Computing: Let $L_{k}^{\text {off }}[n]$ denote the offloaded bits of UE $k \in \mathcal{K}$ to the UAV at slot $n \in \mathcal{N}$. Then the energy consumption can be expressed as

$$
E_{k}^{\text {off }}[n]=P_{k}^{\text {off }}[n] t_{k}^{\text {off }}[n] \equiv \frac{N_{0} t_{k}^{\text {off }}[n]}{h_{k}[n]}\left(2^{\frac{L_{k}^{\text {off }}[n]}{B t_{k}^{\text {off }}[n]}}-1\right),
$$

where $P_{k}^{\text {off }}[n]$ is UE $k$ 's transmit power and $N_{0}$ is the noise power at the UAV ${ }^{2}$. Denote the CPU frequency of the UAV in UE $k$ 's duration $t_{k}[n]$ as $f_{\mathrm{U}, k}[n]$. Thus the completed computation bits and the related energy consumption of the UAV can be respectively expressed as

$$
\begin{aligned}
& L_{\mathrm{U}, k}[n]=t_{k}[n] f_{\mathrm{U}, k}[n] / C_{k}, \\
& E_{\mathrm{U}, k}[n]=t_{k}[n] \kappa_{\mathrm{U}} f_{\mathrm{U}, k}^{3}[n] \equiv \kappa_{\mathrm{U}} C_{k}^{3} t_{k}[n]\left(\frac{L_{\mathrm{U}, k}[n]}{t_{k}[n]}\right)^{3},
\end{aligned}
$$

where $\kappa_{\mathrm{U}}$ is the effective capacitance coefficient of the UAV.

3) Task Offloaded to the AP for Computing: The UAV will further offload part of UEs' offloaded tasks to the more powerful AP for computing. Let $L_{\mathrm{U}, k}^{\mathrm{off}}[n]$ denote UE $k$ 's taskinput bits been offloaded from the UAV to the AP at slot $n$. Thus, the corresponding energy consumption of the UAV for offloading UE $k$ 's task-input data at time slot $n$ is

$$
E_{\mathrm{U}, k}^{\mathrm{off}}[n]=P_{\mathrm{U}, k}^{\mathrm{off}}[n] t_{\mathrm{U}, k}^{\mathrm{off}}[n] \equiv \frac{N_{0} t_{\mathrm{U}, k}^{\mathrm{off}}[n]}{h_{\mathrm{AP}}[n]}\left(2^{\frac{L_{\mathrm{U}, k}^{\mathrm{off}}[n]}{B t_{\mathrm{U}, k}^{\mathrm{oft}}[n]}}-1\right),
$$

where $P_{\mathrm{U}, k}^{\mathrm{off}}[n]$ is the transmit power of the UAV.

During each time slot $n \in \mathcal{N}$, the UAV can only compute or offload the task-input data that has already been received from the UEs. By assuming that the processing delay for decoding and computing preparation at the UAV is one time slot, then we have the following information-causality constraint:

$$
\sum_{i=2}^{n}\left(L_{\mathrm{U}, k}[i]+L_{\mathrm{U}, k}^{\mathrm{off}}[i]\right) \leq \sum_{i=1}^{n-1} L_{k}^{\mathrm{off}}[i]
$$

for $n \in \mathcal{N}_{2}=\{2, \cdots, N\}$ and $k \in \mathcal{K}$. It is easy to see that the UEs should not offload at the last slot, while the UAV should not compute or further offload at the first slot.

\section{Energy Consumption and Harvesting Model}

Except the transmission and computation energy consumption mentioned above, the propulsion energy consumption of the UAV for flying should also be considered, expressed as $E_{\mathrm{U}}^{\mathrm{fy}}[n]=\zeta\|\mathbf{v}[n]\|^{2}$ for $n \in \mathcal{N}$, where $\zeta$ is an efficiency parameter related to UAV's flying energy consumption. Assuming that the AP broadcasts the energy with a constant power $P_{0}$, we can then obtain the harvested energy of the $\mathrm{UAV}$ at each time slot $n \in \mathcal{N}$ as $\widehat{E}_{\mathrm{U}}[n]=\eta_{\mathrm{U}} h_{\mathrm{AP}}[n] P_{0} \tau$, where $0<\eta_{\mathrm{U}} \leq 1$ is the energy conversion efficiency for the UAV. Suppose that the energy transmit power of the UAV at time slot $n$ is $P_{\mathrm{U}}[n]$. Thus the energy consumption of the UAV

\footnotetext{
${ }^{2}$ Without loss of generality, we assume that the noise power at any nodes in the system (the UAV, the AP and all the UEs) is set the same as $N_{0}$.
} 
for WPT and the corresponding harvested energy at UE $k \in \mathcal{K}$ can be respectively expressed as $E_{\mathrm{U}}^{\mathrm{WPT}}[n]=P_{\mathrm{U}}[n] \tau$ and $\widehat{E}_{k}[n]=\eta_{k} h_{k}[n] P_{\mathrm{U}}[n] \tau$ with the energy conversion efficiency $0<\eta_{k} \leq 1$ at slot $n \in \mathcal{N}$.

Combining with the analysis in the above subsections, we can obtain the total energy consumption of UE $k \in \mathcal{K}$ and the UAV in each time slot $n \in \mathcal{N}$ as

$$
\begin{aligned}
& E_{k}[n]=E_{k}^{\mathrm{loc}}[n]+E_{k}^{\mathrm{off}}[n], k \in \mathcal{K}, \\
& E_{\mathrm{U}}[n]=\sum_{k=1}^{K}\left(E_{\mathrm{U}, k}[n]+E_{\mathrm{U}, k}^{\mathrm{off}}[n]\right)+E_{\mathrm{U}}^{\mathrm{fy}}[n]+E_{\mathrm{U}}^{\mathrm{WPT}}[n] .
\end{aligned}
$$

Hence, we can respectively obtain the following energy harvesting causality constraints for UE $k$ and the UAV

$$
\begin{aligned}
& \sum_{i=1}^{n} E_{k}[i] \leq \sum_{i=1}^{n} \widehat{E}_{k}[i], k \in \mathcal{K}, n \in \mathcal{N}, \\
& \sum_{i=1}^{n} E_{\mathrm{U}}[i] \leq \sum_{i=1}^{n} \widehat{E}_{\mathrm{U}}[i], n \in \mathcal{N} .
\end{aligned}
$$

\section{Problem Formulation}

In this paper, we try to maximize the weighted sum completed task-input bits (WSCTB) of UEs by optimizing the UAV's energy transmit power $\left\{P_{\mathrm{U}}[n]\right\}$; UEs' local computing task sizes $\left\{L_{k}^{\text {loc }}[n]\right\}$, offloading task sizes $\left\{L_{k}^{\text {off }}[n]\right\}$; the UAV's computing task sizes $\left\{L_{\mathrm{U}, k}[n]\right\}$, further offloading task sizes $\left\{L_{\mathrm{U}, k}^{\mathrm{off}}[n]\right\}$; along with the UAV's trajectory $\{\mathbf{u}[n]\}$. To this end, the WSCTB maximization problem is formulated as

$$
\begin{aligned}
& \max _{\mathbf{L}, \mathbf{P}_{\mathrm{U}}, \mathbf{u}} \sum_{k=1}^{K} w_{k}\left(\sum_{n=1}^{N} L_{k}^{\text {loc }}[n]+\sum_{n=1}^{N-1} L_{k}^{\text {off }}[n]\right) \\
& \text { s.t. } \sum_{i=2}^{n}\left(L_{\mathrm{U}, k}[i]+L_{\mathrm{U}, k}^{\text {off }}[i]\right) \leq \sum_{i=1}^{n-1} L_{k}^{\text {off }}[i], n \in \mathcal{N}_{2}, k \in \mathcal{K},(14) \\
& \sum_{n=2}^{N}\left(L_{\mathrm{U}, k}[n]+L_{\mathrm{U}, k}^{\text {off }}[n]\right)=\sum_{n=1}^{N-1} L_{k}^{\text {off }}[n], k \in \mathcal{K} \\
& \sum_{i=1}^{n} E_{k}[i](\mathbf{L}, \mathbf{u}) \leq \eta_{k} \tau \sum_{i=1}^{n} h_{k}[i] P_{\mathrm{U}}[i], n \in \mathcal{N}, k \in \mathcal{K} \\
& \sum_{i=1}^{n} E_{\mathrm{U}}[i]\left(\mathbf{L}, \mathbf{P}_{\mathrm{U}}, \mathbf{u}\right) \leq \eta_{\mathrm{U}} \tau P_{0} \sum_{i=1}^{n} h_{\mathrm{AP}}[i], n \in \mathcal{N} \\
& P_{\mathrm{U}}[n] \geq 0, n \in \mathcal{N} \\
& L_{k}^{\text {loc }}[n] \geq 0, n \in \mathcal{N}, k \in \mathcal{K}, \\
& L_{k}^{\text {off }}[N]=0, L_{k}^{\text {off }}[n] \geq 0, n \in \mathcal{N}_{1}, k \in \mathcal{K} \\
& L_{\mathrm{U}, k}[1]=0, L_{\mathrm{U}, k}[n] \geq 0, n \in \mathcal{N}_{2}, k \in \mathcal{K} \\
& L_{\mathrm{U}, k}^{\text {off }}[1]=0, L_{\mathrm{U}, k}^{\text {off }}[n] \geq 0, n \in \mathcal{N}_{2}, k \in \mathcal{K} \\
& \mathbf{u}[0]=\mathbf{u} \mathrm{I}, \mathbf{u}[N]=\mathbf{u}_{\mathrm{F}}, \\
& \|\mathbf{u}[n]-\mathbf{u}[n-1]\| \leq V_{\max } \tau, n \in \mathcal{N},
\end{aligned}
$$

where $\left\{w_{k} \geq 0\right\}_{k \in \mathcal{K}}$ is the set of UEs' weights for the completed task sizes and $\mathcal{N}_{1}=\{1, \cdots, N-1\}$. We denote the optimization variables $\mathbf{L} \triangleq\left\{\mathbf{L}_{k}[n]\right\}_{k \in \mathcal{K}, n \in \mathcal{N}}$ with $\mathbf{L}_{k}[n] \triangleq$ $\left\{L_{k}^{\text {loc }}[n], L_{k}^{\text {off }}[n], L_{\mathrm{U}, k}[n], L_{\mathrm{U}, k}^{\text {off }}[n]\right\}, \mathbf{P}_{\mathrm{U}} \triangleq\left\{P_{\mathrm{U}}[n]\right\}_{n \in \mathcal{N}}$, and $\mathbf{u} \triangleq\{\mathbf{u}[n]\}_{n \in \mathcal{N}}$. In the WSCTB maximization problem, (14c) is the UEs' computation task allocation constraints to make sure that all the UEs' computation task-input data has been computed; (14d) and (14e) present the energy harvesting causality constraints for the UEs and the UAV, respectively.

\section{Algorithm Design}

The formulated problem in (14) is non-convex because of the non-convex constraints (14d) and (14e), where the variables $\mathbf{L}$ and $\mathbf{P}_{\mathrm{U}}$ are strongly coupled with the UAV's trajectory u. In order to properly address these issues, we leverage a twostep block coordinate descending algorithm to optimize the computational task allocation variables in $\mathbf{L}$ and the UAV's energy transmit power in $\mathbf{P}_{\mathrm{U}}$, as well as the UAV's trajectory $\mathbf{u}$ iteratively. The details of the two-step block coordinate descending algorithm for solving the WSCTB maximization problem (14) at the $(\chi+1)$-th $(\chi=0,1,2, \cdots)$ iteration are presented in the following sub-sections.

\section{A. Computation Task and UAV's WPT Power Allocation}

Here, we consider a sub-problem of the original WSCTB maximization problem (14) by fixing the UAV's trajectory $\mathbf{u}$ as the value obtained in the $\chi$-th iteration, i.e., $\mathbf{u}_{\chi}$, denoted as the computation task and UAV's power allocation (CTUPA) problem. Once $\mathbf{u}$ is given, the channels $\left\{h_{k}[n]\right\}_{k \in \mathcal{K}, n \in \mathcal{N}}$ and $\left\{h_{\mathrm{AP}}[n]\right\}_{n \in \mathcal{N}}$ defined in (1) and (2) are known. Hence, the CTUPA problem (P1) can be expressed as

$$
\begin{aligned}
(\mathrm{P} 1): \max _{\mathbf{L}, \mathbf{P}_{\mathrm{U}}} & \sum_{k=1}^{K} w_{k}\left(\sum_{n=1}^{N} L_{k}^{\mathrm{loc}}[n]+\sum_{n=1}^{N-2} L_{k}^{\mathrm{off}}[n]\right) \\
\text { s.t. } & (14 \mathrm{~b})-(14 \mathrm{j}),
\end{aligned}
$$

which is a convex optimization problem with respect to (w.r.t.) $\mathbf{L}$ and $\mathbf{P}_{\mathrm{U}}$. In order to gain more insights of the solution, we leverage the Lagrange method [15] to solve problem (P1), and the optimal solution is given in the following theorem.

Theorem 1. The optimal solution of problem (P1) related to $U E k \in \mathcal{K}$ is given in (16)-(20) at the top of the next page, where $\gamma_{k, n}^{*} \geq 0, \xi_{k, n}^{*} \geq 0$ and $\rho_{n}^{*} \geq 0$ for $k \in \mathcal{K}, n \in \mathcal{N}$ are respectively the optimal Lagrange multipliers associated with the inequality constraints (14b), (14d) and (14e) in problem $(P 1)$, while $\lambda_{k}^{*}$ for $k \in \mathcal{K}$ are the optimal Lagrange multipliers associated with the equality constraints in (14c).

\section{Proof. See Appendix A.}

The optimal Lagrange multipliers of the inequality constraints (14b), (14d), (14e) can be obtained by a subgradientbased algorithm, while those related to the equality constraints (14c) can be obtained by the bi-section search method.

\section{B. UAV Trajectory Design}

In this section, a sub-problem for designing the UAV's trajectory $\mathbf{u}$ is considered by assuming that $\mathbf{L}$ and $\mathbf{P}_{U}$ are fixed as the optimized values obtained in the $(\chi+1)$-th iteration through Theorem 1 . Updating UE $k$ 's offloading bits at time slot $n$ as a function of $\mathbf{u}[n]$ for $k \in \mathcal{K}, n \in \mathcal{N}_{1}$ as

$$
L_{k}^{\text {off }}[n](\mathbf{u}[n])=t_{k, \chi+1}^{\text {off }}[n] B \log _{2}\left(1+\frac{P_{k}^{\text {off }}[n] h_{0} / N_{0}}{\left\|\mathbf{u}[n]-\mathbf{v}_{k}\right\|^{2}+H^{2}}\right) .
$$

Hence, the UAV trajectory design problem (P2) is written as

$$
\begin{aligned}
(\mathrm{P} 2): \max _{\mathbf{u}} & \sum_{k=1}^{K} w_{k} \sum_{n=1}^{N-1} L_{k}^{\text {off }}[n](\mathbf{u}[n]) \\
\text { s.t. } & (14 \mathrm{~d}),(14 \mathrm{e}),(14 \mathrm{k}),(141) .
\end{aligned}
$$




$$
\begin{aligned}
& L_{k}^{\text {loc* }}[n]=\frac{\tau}{C_{k}} \sqrt{\frac{w_{k}}{3 \kappa_{k} C_{k} \sum_{i=n}^{N} \xi_{k, i}^{*}}}, n \in \mathcal{N}, \\
& L_{k}^{\text {off } *}[n]=\left\{\begin{array}{l}
B t_{k}^{\text {off }}[n]\left[\log _{2}\left[B h_{k}[n]\left(w_{k}+\lambda_{k}^{*}+\sum_{i=n+1}^{N-1} \gamma_{k, i}^{*}\right)\right]^{+}-\log _{2}\left(N_{0} \ln 2 \sum_{i=n}^{N} \xi_{k, i}^{*}\right)\right]^{+}, n \in \mathcal{N}_{1}, \\
0, \quad n=N,
\end{array}\right. \\
& L_{\mathrm{U}, k}^{*}[n]=\left\{\begin{array}{l}
t_{k}[n] \sqrt{\frac{1}{3 \kappa_{\mathrm{U}} C_{k}^{3} \sum_{i=n}^{N} \rho_{i}}\left[-\sum_{i=n}^{N-1} \gamma_{k, i}^{*}-\lambda_{k}^{*}\right]^{+}}, n \in \mathcal{N}_{2}, \\
0, \quad n=1,
\end{array}\right. \\
& L_{\mathrm{U}, k}^{\mathrm{off} *}[n]=\left\{\begin{array}{l}
B t_{\mathrm{U}, k}^{\mathrm{off}}[n]\left[\log _{2}\left[B h_{\mathrm{AP}}[n]\left(-\sum_{i=n}^{N-1} \gamma_{k, i}^{*}-\lambda_{k}^{*}\right)\right]^{+}-\log _{2}\left(N_{0} \ln 2 \sum_{i=n}^{N} \rho_{i}\right)\right]^{+}, n \in \mathcal{N}_{2}, \\
0, \quad n=1,
\end{array}\right. \\
& P_{\mathrm{U}}^{*}[n]=\left\{\begin{array}{l}
P_{\mathrm{U}}^{\text {low }}[n], \quad \text { if } \sum_{i=n}^{N} \rho_{i}^{*}>\sum_{k=1}^{K}\left(\eta_{k} h_{k}[n] \sum_{i=n}^{N} \xi_{k, i}^{*}\right), n \in \mathcal{N} \\
P_{\mathrm{U}}^{\mathrm{up}}[n], \quad \text { otherwie, } n \in \mathcal{N},
\end{array}\right.
\end{aligned}
$$

where $P_{\mathrm{U}}^{\text {low }}[n]=\max \left\{\left[\frac{\sum_{i=1}^{n} E_{k}[i]\left(\mathbf{L}^{*}, \mathbf{u}\right)}{\eta_{k} \tau h_{k}[n]}-\frac{\sum_{i=1}^{n-1} h_{k}[i] P_{\mathrm{U}}[i]}{h_{k}[n]}\right]^{+}\right\}_{k \in \mathcal{K}}$ and $P_{\mathrm{U}}^{\mathrm{up}}[n]=\left[\frac{1}{\tau} \sum_{i=1}^{n}\left(\eta_{\mathrm{U}} \tau P_{0} h_{\mathrm{AP}}[i]-E_{\mathrm{U}}^{\text {self }}[i]\left(\mathbf{L}^{*}, \mathbf{u}\right)\right)-\sum_{i=1}^{n-1} P_{\mathrm{U}}[i]\right]^{+}$ with $E_{\mathrm{U}}^{\text {self }}[n]\left(\mathbf{L}^{*}, \mathbf{u}\right)=\sum_{k=1}^{K}\left(E_{\mathrm{U}, k}[n]+E_{\mathrm{U}, k}^{\mathrm{off}}[n]\right)+E_{\mathrm{U}}^{\mathrm{fly}}[n]$ in (20) denote a lower bound and a upper bound solution of $P_{\mathrm{U}}[n]$, respectively.

Note that the objective function is not concave and the constraints (14d), (14e) are not convex because of the non-concave items $\left\{L_{k}^{\text {off }}[n](\mathbf{u}[n])\right\}$ and $\left\{h_{k}[n](\mathbf{u}[n])\right\},\left\{h_{\mathrm{AP}}[n](\mathbf{u}[n])\right\}$. To address this issue, we leverage the successive convex approximation (SCA) algorithm to obtain an efficient solution of problem $(\mathrm{P} 2)$. In the $(m+1)$-th $(m=0,1,2, \cdots)$ iteration, the non-concave item $L_{k}^{\text {off }}[n](\mathbf{u}[n])$ in the objective function is approximated by its concave lower bound as follows

$$
\begin{aligned}
& L_{k}^{\text {off }}[n](\mathbf{u}[n]) \geq \widetilde{L}_{k}^{\text {off }}[n](\mathbf{u}[n]) \\
= & t_{k}^{\text {off }}[n] B\left\{\log _{2}\left(1+\frac{P_{k}^{\text {off }}[n] h_{0} / N_{0}}{d_{k}^{2}\left(\mathbf{u}_{m}[n]\right)}\right)-\right. \\
& \left.\frac{P_{k}^{\text {off }}[n] h_{0}}{N_{0} \ln 2} \frac{\left\|\mathbf{u}[n]-\mathbf{v}_{k}\right\|^{2}-\left\|\mathbf{u}_{m}[n]-\mathbf{v}_{k}\right\|^{2}}{d_{k}^{2}\left(\mathbf{u}_{m}[n]\right)\left(d_{k}^{2}\left(\mathbf{u}_{m}[n]\right)+P_{k}^{\text {off }}[n] h_{0} / N_{0}\right)}\right\},
\end{aligned}
$$

which is obtained through the first-order Taylor expansion of $L_{k}^{\text {off }}[n](\mathbf{u}[n])$ around the optimized point at the $m$-th iteration, denoted as $\mathbf{u}_{m}[n]$, w.r.t. the convex item $\left\|\mathbf{u}[n]-\mathbf{v}_{k}\right\|^{2}$. We define $d_{k}^{2}\left(\mathbf{u}_{m}[n]\right)=\left\|\mathbf{u}_{m}[n]-\mathbf{v}_{k}\right\|^{2}+H^{2}$. Similarly, $\eta_{\mathrm{U}} \tau P_{0} h_{\mathrm{AP}}[n]$ and $\eta_{k} \tau P_{\mathrm{U}}[n] h_{k}[n]$ in the constraints (14d), (14e) are approximated by their concave lower bounds below

$$
\begin{aligned}
\eta_{\mathrm{U}} \tau P_{0} h_{\mathrm{AP}}[n] & \geq \eta_{\mathrm{U}} \tau P_{0} \widetilde{h}_{\mathrm{AP}}[n], n \in \mathcal{N}, \\
\eta_{k} \tau P_{\mathrm{U}}[n] h_{k}[n] & \geq \eta_{k} \tau P_{\mathrm{U}}[n] \widetilde{h}_{k}[n], k \in \mathcal{K}, n \in \mathcal{N},
\end{aligned}
$$

where $\widetilde{h}_{\mathrm{AP}}[n]=h_{0} \frac{2\left\|\mathbf{u}_{m}[n]-\mathbf{v}_{0}\right\|^{2}+H^{2}-\left\|\mathbf{u}[n]-\mathbf{v}_{0}\right\|^{2}}{\left(\left\|\mathbf{u}_{m}[n]-\mathbf{v}_{0}\right\|^{2}+H^{2}\right)^{2}}$ and $\widetilde{h}_{k}[n]=$ $h_{0} \frac{2\left\|\mathbf{u}_{m}[n]-\mathbf{v}_{k}\right\|^{2}+H^{2}-\left\|\mathbf{u}[n]-\mathbf{v}_{k}\right\|^{2}}{\left(\left\|\mathbf{u}_{m}[n]-\mathbf{v}_{k}\right\|^{2}+H^{2}\right)^{2}}$. The approximated problem of (P2) in the $(m+1)$-th iteration is convex w.r.t. the UAV's trajectory $\mathbf{u}$, and we use the software CVX [16] to solve it.

\section{Simulation Results}

In this section, simulation results are given to show the UAV's trajectory and the performance of the proposed algo-
TABLE I

SIMULATION PARAMETERS

\begin{tabular}{|l|l|l|}
\hline Parameter & Symbol & Value \\
\hline System bandwidth & $B$ & $40 \mathrm{MHz}$ \\
\hline Task completion time & $T$ & 5 seconds \\
\hline Number of time slots & $N$ & 50 \\
\hline Number of ground UEs & $K$ & 4 \\
\hline The channel power gain & $h_{0}$ & $-30 \mathrm{~dB}$ \\
\hline The noise power & $N_{0}$ & $-60 \mathrm{dBm}$ \\
\hline The AP's WPT power & $P_{0}$ & $150 \mathrm{dBm}$ \\
\hline Altitude of the UAV & $H$ & $10 \mathrm{~m}$ \\
\hline UAV's maximum speed & $V_{\max }$ & $20 \mathrm{~m} / \mathrm{s}$ \\
\hline UAV's flying energy efficiency & $\zeta$ & 0.2 \\
\hline The switched capacitance & $\kappa_{\mathrm{U}}, \kappa_{k}(k \in \mathcal{K})$ & $10^{-28}$ \\
\hline Required CPU cycles per bit & $C_{k}(k \in \mathcal{K})$ & $1000 \mathrm{cycles} / \mathrm{bit}$ \\
\hline
\end{tabular}

rithms. The basic simulation parameters are listed in Table I unless specified otherwise, and the UEs' horizontal locations are set as $\left[\mathbf{v}_{1}, \mathbf{v}_{2}, \mathbf{v}_{3}, \mathbf{v}_{4}\right]=[(10,10),(-10,10),(-10,-10)$, $(10,-10)]$ in the following figures.

Fig. 2 depicts the UAV's trajectory under different scenarios with varied initial and final locations and UEs' weights for the completed task size. The results show that the WSCTB of UEs are highly depends on the UEs' weights of the completed task size, i.e., w. The UAV tends to fly close to the UEs with lager weights so as to improve the WSCTB of UEs.

In Fig. 3, the performance of the proposed solution is presented in comparison with two baselines: the 'Direct trajectory' and 'Semi-circle trajectory', denoting two schemes with the specified given trajectory. The WSCTB of UEs w.r.t. the time duration $T$ and the number of time slots $N$ are depicted in (a) and (b), respectively, in the case of $\mathbf{w}=[0.7,0.1,0.1,0.1]$. 

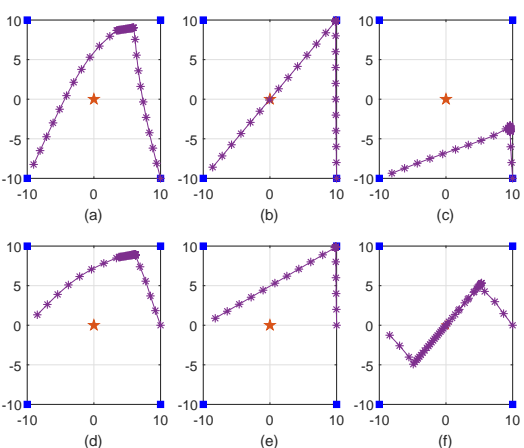

Fig. 2. The trajectories of the UAV with different initial and final locations of the UAV and different weight vectors for the UEs: $\mathbf{u}_{\mathrm{I}}=(-10,-10)$, $\mathbf{u}_{\mathrm{F}}=(10,-10)$ for (a), (b), (c); $\mathbf{u}_{\mathrm{I}}=(-10,0), \mathbf{u}_{\mathrm{F}}=(10,0)$ for (d), (e), (f); $\mathbf{w}=[0.4,0.3,0.2,0.1]$ for (a), (d); $\mathbf{w}=[0.7,0.1,0.1,0.1]$ for (b), (e); $\mathbf{w}=[0.4,0.1,0.1,0.4]$ for $(\mathrm{c}) ; \mathbf{w}=[0.4,0.1,0.4,0.1]$ for $(\mathrm{f})$
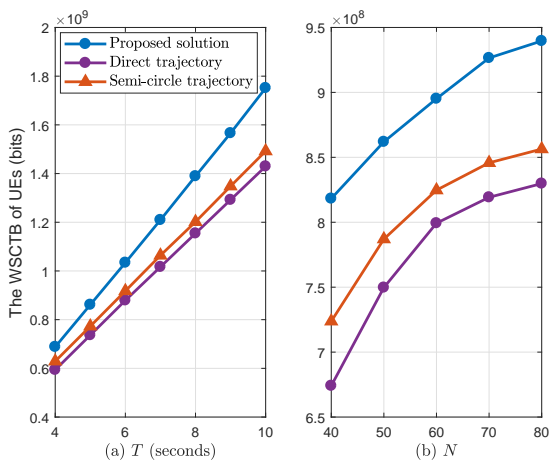

Fig. 3. The WSCTB of the UEs w.r.t. the time duration: $T$ in (a) with $N=50$ and the number of time slots $N$ in (b) with $T=5 \mathrm{~s}$.

It is easy to see that the proposed solution can achieve significant performance improvement compared with the two schemes without trajectory design, indicating the importance of the UAV's trajectory optimization.

\section{CONCLUSION}

A wireless-powered UAV-assisted MEC architecture is investigated in this paper, where the UAV serves as an energy relay, an information relay as well as a MEC server to provide the UEs with sustainable energy supply and help them offload or compute tasks. The WSCTB of the UEs is maximized by jointly optimizing the UE's task allocation, the UAV's energy transmit power and the trajectory. The simulation results show that the proposed algorithm can achieve great performance improvement in comparison with two basedlines.

\section{ACKNOWLEDGMENT}

This work is supported by the U.K. EPSRC under Grants EP/K015893/1 and EP/L026031/1.

\section{ApPendix A: Proof of Theorem 1}

The task allocation parameters $L_{k}^{\text {loc }}[n], L_{k}^{\text {off }}[n], L_{\mathrm{U}, k}[n]$ and $L_{\mathrm{U}, k}^{\mathrm{off}}[n]$ given in Theorem 1 can be obtained by leveraging the Lagrange duality method in company with the KarushKuhn-Tucker (KKT) conditions [15]. With the obtained $\mathbf{L}^{*}$, the optimization of the UAV's energy transmit power $\mathbf{P}_{\mathrm{U}}[n]$ is a liner programming. It is clear that a lower bound of $P_{\mathrm{U}}[n]$ can be obtained from the constraints in (14d), i.e.,

$P_{\mathrm{U}}[n] \geq \max \left\{\frac{\sum_{i=1}^{n} E_{k}[i]\left(\mathbf{L}^{*}, \mathbf{u}\right)}{\eta_{k} \tau h_{k}[n]}-\frac{\sum_{i=1}^{n-1} h_{k}[i] P_{\mathrm{U}}[i]}{h_{k}[n]}\right\} \triangleq P_{k \in \mathcal{K}}^{\mathrm{low}}[n]$.

Also, a upper bound of $P_{\mathrm{U}}[n]$ can be obtained from (14e) as

$$
\begin{aligned}
& P_{\mathrm{U}}[n] \leq \frac{1}{\tau} \sum_{i=1}^{n}\left[\eta_{\mathrm{U}} \tau P_{0} h_{\mathrm{AP}}[i]-E_{\mathrm{U}}^{\mathrm{fy}}[i]-\right. \\
& \left.\sum_{k=1}^{K}\left(E_{\mathrm{U}, k}[i]+E_{\mathrm{U}, k}^{\mathrm{off}}[i]\right)\right]-\sum_{i=1}^{n-1} P_{\mathrm{U}}[i] \triangleq P_{\mathrm{U}}^{\mathrm{up}}[n],
\end{aligned}
$$

Hence, we can obtain the solution of $\left\{P_{\mathrm{U}}[n]\right\}$ in (20) through the KKT conditions by using the Lagrange method.

\section{REFERENCES}

[1] P. Mach and Z. Becvar, "Mobile edge computing: A survey on architecture and computation offloading," IEEE Commun. Surveys Tuts., vol. 19, no. 3, pp. 1628-1656, thirdquarter 2017.

[2] T. Q. Dinh, J. Tang, Q. D. La, and T. Q. S. Quek, "Offloading in mobile edge computing: Task allocation and computational frequency scaling," IEEE Trans. Commun., vol. 65, no. 8, pp. 3571-3584, Aug. 2017.

[3] C. You, K. Huang, H. Chae, and B. H. Kim, "Energy-efficient resource allocation for mobile-edge computation offloading," IEEE Trans. Wireless Commun., vol. 16, no. 3, pp. 1397-1411, Mar. 2017.

[4] T. X. Tran and D. Pompili, "Joint task offloading and resource allocation for multi-server mobile-edge computing networks," IEEE Trans. Veh. Technol., vol. 68, no. 1, pp. 856-868, Jan. 2019.

[5] X. Hu, L. Wang, K.-K. Wong, Y. Zhang, Z. Zheng, and M. Tao, "Edge and central cloud computing: A perfect pairing for high energy efficiency and low-latency," arXiv preprint arXiv:1806.08943, 2018.

[6] C. You, K. Huang, and H. Chae, "Energy efficient mobile cloud computing powered by wireless energy transfer," IEEE J. Sel. Areas Commun., vol. 34, no. 5, pp. 1757-1771, May 2016.

[7] X. Hu, K. Wong, and K. Yang, "Wireless powered cooperation-assisted mobile edge computing," IEEE Trans. Wireless Commun., vol. 17, no. 4, pp. 2375-2388, Apr. 2018.

[8] Y. Mao, J. Zhang, and K. B. Letaief, "Dynamic computation offloading for mobile-edge computing with energy harvesting devices," IEEE $J$. Sel. Areas Commun., vol. 34, no. 12, pp. 3590-3605, Dec. 2016.

[9] S. Jeong, O. Simeone, and J. Kang, "Mobile edge computing via a UAVmounted cloudlet: Optimization of bit allocation and path planning," IEEE Trans. Veh. Technol., vol. 67, no. 3, pp. 2049-2063, Mar. 2018.

[10] X. Hu, K.-K. Wong, K. Yang, and Z. Zheng, "UAV-assisted relaying and edge computing: Scheduling and trajectory optimization," arXiv preprint arXiv:1812.02658, 2018

[11] X. Cao, J. Xu, and R. Zhang, "Mobile edge computing for cellularconnected UAV: Computation offloading and trajectory optimization," in proc. IEEE SPAWC, Kalamate, Greece, Jun. 2018.

[12] Y. Zeng, R. Zhang, and T. J. Lim, "Wireless communications with unmanned aerial vehicles: opportunities and challenges," IEEE Commun. Mag., vol. 54, no. 5, pp. 36-42, May 2016.

[13] F. Zhou, Y. Wu, R. Q. Hu, and Y. Qian, "Computation rate maximization in UAV-enabled wireless-powered mobile-edge computing systems," IEEE J. Sel. Areas Commun., vol. 36, no. 9, pp. 1927-1941, Sep. 2018.

[14] T. D. Burd and R. W. Brodersen, "Processor design for portable systems," J. VLSI Signal Process. Syst., vol. 13, no. 2-3, pp. 203-221, 1996.

[15] S. Boyd and L. Vandenberghe, Convex optimization. Cambridge university press, 2004.

[16] M. Grant, S. Boyd, and Y. Ye, "CVX: Matlab software for disciplined convex programming," 2008. 